\title{
Lincomycin induces melanogenesis through the activation of MITF via p38 MAPK, AKT, and PKA signaling pathways
}

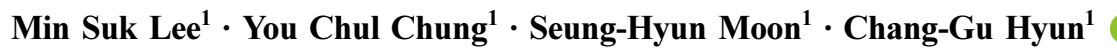 \\ Received: 21 August 2021 / Accepted: 17 September 2021 / Published Online: 31 December 2021 \\ (C) The Korean Society for Applied Biological Chemistry 2021
}

\begin{abstract}
Lincomycin is a lincosamide antibiotic isolated from the actinomycete Streptomyces lincolnensis. Moreover, it has been found to be effective against infections caused by Staphylococcus, Streptococcus, and Bacteroides fragillis. To identify the melanininducing properties of lincomycin, we used B16F10 melanoma cells in this study. The melanin content and intracellular tyrosinase activity in the cells were increased by lincomycin, without any cytotoxicity. Western blot analysis indicated that the protein expressions of tyrosinase, tyrosinase related protein 1 (TRP1) and TRP2 increased after lincomycin treatment. In addition, lincomycin enhanced the expression of master transcription regulator of melanogenesis, a microphthalmia-associated transcription factor (MITF). Lincomycin also increased the phosphorylation of p38 mitogen-activated protein kinase (MAPK) and decreased the AKT phosphorylation. Moreover, the activation of tyrosinase activity by lincomycin was inhibited by the treatment with SB203580, which is p38 inhibitor. Furthermore, we also found that lincomycininduced tyrosinase expression was reduced by $\mathrm{H}-89$, a specific protein kinase A (PKA) inhibitor. These results indicate that lincomycin stimulate melanogenesis via MITF activation via p38 MAPK, AKT, and PKA signal pathways. Thus, lincomycin can potentially be used for treatment of hypopigmentation disorders.
\end{abstract}

Keywords AKT - Lincomycin · Melanogenesis · Mitogenactivated protein kinase $\cdot \mathrm{p} 38 \cdot$ Protein kinase A

Min Suk Lee and You Chul Chung are contributed equally to this work

Chang-Gu Hyun $(\varangle)$

E-mail: cghyun@jejunu.ac.kr

${ }^{1}$ Jeju Inside Agency and Cosmetic Science Center, Department of Chemistry and Cosmetics, Jeju National University, Jeju 63243, Republic of Korea

This is an Open Access article distributed under the terms of the Creative Commons Attribution Non-Commercial License (http://creativecommons. org/licenses/by-nc/3.0/) which permits unrestricted non-commercial use, distribution, and reproduction in any medium, provided the original work is properly cited.

\section{Introduction}

Melanogenesis is a defense mechanism against irritation caused by UV radiation, environmental pollution, and other factors. Melanin is a pigment produced by melanosomes in melanocytes. Melanocytes are located in the basal layer of the mammalian epidermis and deliver melanosomes to the surrounding keratinocytes, where they play important roles in skin protection from harmful UV radiation by scavenging free radicals or dispersing the incoming UV light [1-6].

Well-known enzymes related to melanogenesis include tyrosinase, tyrosinase related protein (TRP) 1 , and 2 . Tyrosinase is a catalytic enzyme involved in determining the initial rate in the melanogenesis and plays a very important role in the entire melanin synthesis mechanism. Tyrosinase, which acts tyrosine hydroxylase or DOPA-oxidase, binds a hydroxyl group to tyrosine and converts it to 3,4-dihydroxyphenyl-L-alanin (L-DOPA; diphenol), and oxidizes DOPA into dopaquinone. Dopaquinone takes intramolecular cyclization by tyrosinase to form leucoDOPAchrome, which is oxidized to DOPAchrome. DOPAchrome converted from Dopaquinon is converted to 5,6-dihydroxy-2-indolylcarboxylic acid (DHICA) by TRP2. DHICA is then transformed into indole5,6-quinone-carboxylic acid by TRP1 to finally produce black or brown eumelanin [7-10].

Microphthalmia-associated transcription factor (MITF) plays an important role in melanin synthesis via regulating expression of melanogenic enzymes (tyrosinase, TRP1, TRP2). The main role of MITF is to increase the expression of melanogenic enzymes by binding to the tyrosinase promoter, which is called the M-box. Accordingly, recent studies have indicated that hypopigmentation is prevented by the activation of MITF expression [11,12].

Phosphorylation of mitogen-activated protein kinases (MAPK) family, which consist of p38 MAPK, extracellular signal-regulated kinase (ERK), and c-Jun N-terminal kinase (JNK), are closely related to MITF regulation. Recent studies have reported that the activation of $\mathrm{p} 38$ and JNK, and the inhibition of ERK phosphorylation stimulate MITF and melanogenic enzymes expression, which up- 
regulates to melanogenesis [13-15].

In addition, it has been reported that the activation of protein kinase A (PKA) induces MITF expression. PKA is activated via the cyclic adenosine monophosphate (cAMP) pathway and is transferred to the nucleus of melanocytes, increasing the phosphorylation of cAMP response element binding protein (CREB). This increases the expression of MITF, which results in the activation of melanogenesis [16-18]. Other reports indicate that the inhibition of the AKT (protein kinase B) signaling pathway upregulates melanogenesis by increasing the MITF level, and subsequently, melanogenic enzymes expression [19-22].

Lincomycin, isolated from Streptomyces lincolnensis, is a lincosamide-based antibiotic. lincomycin was released for medical use in September 1964. Lincomycin is commonly used to treat infections caused by gram-positive bacteria, such as Staphylococcus, Streptococcus, and Bacteroides fragilis [23,24]. Although lincomycin has been utilized clinically for a long time, it causes several side effects (rash, diarrhea, and vomiting due to an allergic reaction), leading to the introduction of safer antibiotics, such as clindamycin [25]. Therefore, we performed an experiment focusing on new efficacy of lincomycin. Recent studies have reported that several antibiotics isolated from Streptomyces species, such as tobramycin, fosfomycin (melanogenesis stimulator), and kanamycin (melanogenesis inhibitor), are related to melanogenesis [26-28]. However, the effect of lincomycin on melanogenesis has not yet been investigated. Therefore, in this study, mouse B16F10 melanoma cells were treated with lincomycin to examine its effects on melanogenesis.

\section{Materials and Methods}

\section{Chemicals and reagents}

Lincomycin hydrochloride monohydrate was obtained from Tokyo Chemical Industry Co., Ltd. (Seoul, Korea). L-DOPA, $\alpha-$ melanocyte stimulating hormone, H-89 (PKA inhibitor), and $\mathrm{NaOH}$ were purchased from Sigma-Aldrich (St. Louis, MO, USA). Primary antibodies against MITF, tyrosinase, tyrosinase related protein-1, 2, and $\beta$-actin were purchased from Santa Cruz Biotechnology (Dallas, TX, USA). Primary antibodies against phospho-AKT, AKT, phospho-p38, p38, and secondary antibody of anti-rabbit and anti-mouse IgG HRP-linked were procured from Cell Signaling Technology (Danvers, MA, USA). p38 inhibitor (SB203580) was purchased from Cayman Chemical (Ann Arbor, MI, USA). Radioimmunoprecipitation assay (RIPA) buffer and enhanced chemiluminescence (ECL) were purchased from Biosesang (Seongnam, Gyeonggi-do, Korea). 2×Laemmli sample buffer was obtained from Bio-Rad Laboratories (Hercules, CA, USA).

\section{Cell culture}

B16F10 mouse melanoma and HaCaT human keratinocyte cells were purchased from ATCC (The Global Bioresource Center). Both B16F10 and HaCaT cells were cultured in Dulbecco's Modified Eagle Medium (DMEM) containing 10\% fetal bovine serum (FBS) and $1 \%$ penicillin/streptomycin $(100 \mu \mathrm{g} / \mathrm{mL})$ at 37 ${ }^{\circ} \mathrm{C}$, and $5 \% \mathrm{CO}_{2}$, and were sub-cultured every 3 days.

\section{Measurement of cell viability}

B16F10 melanoma cells $\left(0.8 \times 10^{4}\right.$ cells/well $)$ were seeded and pre-incubated for $24 \mathrm{~h}$, and then treated with lincomycin $(0.25$, $0.5,1,2$, and $4 \mathrm{mg} / \mathrm{mL}$ ) for $72 \mathrm{~h}$. Then, cells were treated with MTT reagent, diluted to $0.2 \mathrm{mg} / \mathrm{mL}$, for $4 \mathrm{~h}$, after which the formazan crystals were dissolved in diethyl sulfoxide (DMSO). The absorbance of the each well was determined at $540 \mathrm{~nm}$ using a microplate reader (Tecan, Mannedorf, Switzerland).

HaCaT cells $\left(0.8 \times 10^{5}\right.$ cells/well $)$ were seeded and pre-incubated for $24 \mathrm{~h}$. The supernatant was removed and the cells were cultured in FBS-free DMEM with lincomycin $(0.5,1,2$, and $4 \mathrm{mg} / \mathrm{mL})$ for $24 \mathrm{~h}$. Then, after treating cells with the MTT solution $(0.2 \mathrm{mg} /$ $\mathrm{mL}$ ) for $4 \mathrm{~h}$, after which the formazan crystals was dissolved in DMSO. The absorbance of the each well was determined at 540 $\mathrm{nm}$ using a microplate reader.

\section{Measurement of intracellular melanin content}

B16F10 melanoma cells were seeded in a 6 -well plate at $0.8 \times 10^{5}$ cells/well, and incubated for $24 \mathrm{~h}$. The cells were then treated with lincomycin $(0.25,0.5,1$, and $2 \mathrm{mg} / \mathrm{mL})$ and $\alpha-\mathrm{MSH}(200 \mathrm{nM})$ for 72 h. $\alpha$-MSH served as the positive control. After the supernatant was removed, the cells were washed twice with phosphate buffered saline (PBS), and then the cell pellets were collected and dissolved in $1 \mathrm{M} \mathrm{NaOH}$ for $1 \mathrm{~h}$ at $70{ }^{\circ} \mathrm{C}$. Each cell lysate was transferred to a 96-well plate and the absorbance of the each well was determined at $540 \mathrm{~nm}$ using a spectrophotometer.

\section{Measurement of tyrosinase activity}

B16F10 melanoma cells were seeded into $60-\mathrm{mm}$ dishes at $0.8 \times 10^{5}$ cells/dish and pre-incubated for $24 \mathrm{~h}$. Subsequently, the B16F10 cells were treated with lincomycin $(0.25,0.5,1$, and 2 $\mathrm{mg} / \mathrm{mL})$ and $\alpha-\mathrm{MSH}(200 \mathrm{nM})$ for $72 \mathrm{~h}$ at $37{ }^{\circ} \mathrm{C}$ and $5 \% \mathrm{CO}_{2}$ under humidified conditions. After the media was removed, cells were washed twice with PBS and the cell pellets were collected and lysed in RIPA buffer containing a $1 \%$ protease inhibitor cocktail. After centrifugation for $15 \mathrm{~min}$ at $13,000 \mathrm{rpm}$, the supernatant was collected, and the protein level of each lysate was quantified using the bicinchoninic acid (BCA) kit. Next, $20 \mu \mathrm{L}$ of each adjusted protein sample was mixed with $80 \mu \mathrm{L}$ of L-DOPA $(2 \mathrm{mg} / \mathrm{mL})$ in a $96-w e l l$ plate. After incubation at $37{ }^{\circ} \mathrm{C}$ for $2 \mathrm{~h}$, the absorbance was measured at $490 \mathrm{~nm}$ using a microplate reader.

\section{Western blot analysis}

B16F10 cells were seeded into $60-\mathrm{mm}$ dishes and cultured in media containing $10 \% \mathrm{FBS}$ and $1 \%$ penicillin/streptomycin. After 
pre-incubation, the cells were treated with various concentrations of lincomycin and $\alpha$-MSH. Subsequently, the supernatant was removed and cells were washed twice with PBS, and then the cell pellets were collected into $1.5 \mathrm{~mL}$ microtubes, followed by lysis in RIPA buffer containing a $1 \%$ protease inhibitor cocktail. After centrifugation for $20 \mathrm{~min}$ at $13,000 \mathrm{rpm}$, the supernatant was collected, and the protein level of each lysate was quantified using the BCA kit. The adjusted lysate and $2 \times$ Laemmli sample buffer $(1: 1)$ were mixed to obtain an equal amount of protein $(20 \mu \mathrm{g})$ in each sample. After heating the samples to $95{ }^{\circ} \mathrm{C}$ for $6 \mathrm{~min}$, equal volumes of the sample $(20 \mu \mathrm{L})$ were loaded on $10 \%$ sodium dodecyl sulfate polyacrylamide gels. The proteins, which were separated by weight, were transferred to polyvinylidene difluoride membranes, and the membranes were blocked using TBST containing 5\% skim milk for $2 \mathrm{~h}$. Then, the membranes were washed six times with Tris-buffered saline $(20 \mathrm{mM}$ Tris base, $137 \mathrm{mM} \mathrm{NaCl}, \mathrm{pH} 7.6$ ) containing $0.1 \%$ Tween 20 solution (TBST), every $5 \mathrm{~min}$, for $30 \mathrm{~min}$. Afterward, the membranes were incubated overnight at $4{ }^{\circ} \mathrm{C}$ with specific primary antibodies diluted in TBST $(1: 1,000)$. After incubation, the membranes were washed six times with TBST every $5 \mathrm{~min}$ and incubated with HRP-linked secondary antibodies (IgG, 1:3,000) for $2 \mathrm{~h}$, followed by washing six times with TBST. The target protein expression levels were visualized using an ECL kit.

\section{Statistical analysis}

All data are expressed as the mean \pm standard deviation (SD: $n=4)$. Each experiment was repeated at least four times. The statistical analyses were determined by one-way ANOVA using SPSS (v. 22.0, SPSS Inc., Chicago, IL, USA). Values of $p<0.05$

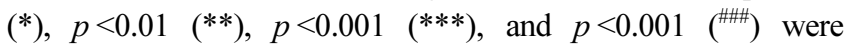
considered statistically significant difference.

\section{Results}

\section{Effect of lincomycin on cell viability of cells}

We first to determine whether lincomycin has cytotoxicity against B16F10 cells, a MTT assay was performed. Briefly, the B16F10 cells were treated with various concentrations of lincomycin $(0.25$, $0.5,1,2$, and $4 \mathrm{mg} / \mathrm{mL}$ ) for $72 \mathrm{~h}$. As a result, there were no significant effects on cell viability of B16F10 cells treated with lincomycin (concentration: 0.25 to $2 \mathrm{mg} / \mathrm{mL}$ ), relative to the untreated control cells (Fig. 1B). However, the cell viability

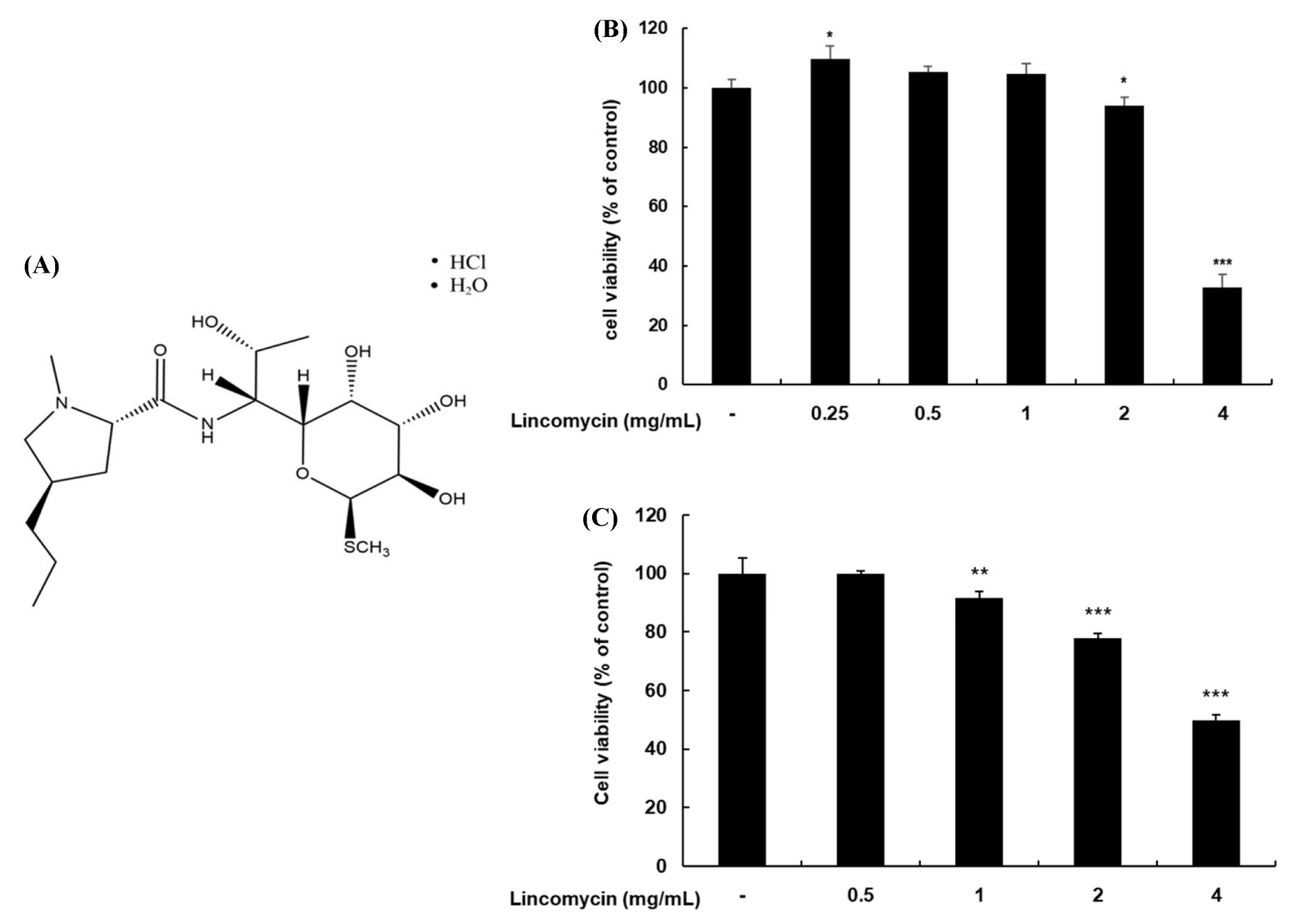

Fig. 1 The cell viability of lincomycin on B16F10 and HaCaT cells. (A) Structure of lincomycin. The cells were treated with lincomycin $(0.25,0.5,1$, 2, and $4 \mathrm{mg} / \mathrm{mL}$ ) for $72 \mathrm{~h}$, and cell viability on (B) B16F10 and (C) HaCaT cells were determined using a MTT assay. The results of all data are expressed as the mean \pm standard deviation (SD: $\mathrm{n}=4$ ) of at least four independent experiments $(\mathrm{n}=4)$. ${ }^{*} p<0.05,{ }^{* * *} p<0.001$ vs. untreated control cells 


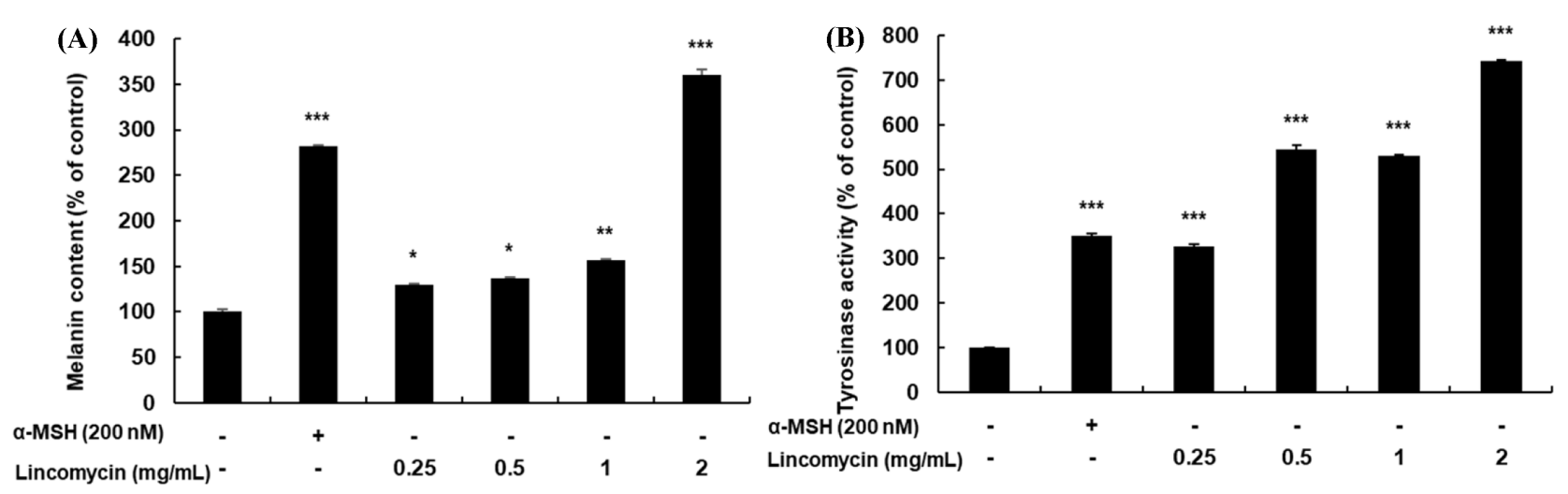

Fig. 2 The effect of lincomycin on melanin content and tyrosinase activity in B16F10 melanoma cells. The cells were treated with various concentrations of lincomycin for $72 \mathrm{~h}$. $\alpha-\mathrm{MSH}$ was used as the positive control. (A) Melanin content and (B) tyrosinase activity are expressed as percentages compared to the untreated control cells. The results of all data are expressed as the mean \pm standard deviation $(\mathrm{SD}$ : $\mathrm{n}=4)$ of at least four independent experiments. ${ }^{*} p<0.05,{ }^{*} p<0.01,{ }^{* * *} p<0.001$ vs. untreated control cells. $\alpha$-MSH: positive control

decreased by $63 \%$ in the group treated with $4 \mathrm{mg} / \mathrm{mL}$ lincomycin. Therefore, we performed subsequent experiments using only the following lincomycin concentrations: $0.25,0.5,1$, and $2 \mathrm{mg} / \mathrm{mL}$. In addition, melanocytes are dendritic cells found in the base layer of the epidermis of the skin, producing melanosomes, and transferring them to the surrounding keratinocytes [29-32]. Therefore, a cell viability test was performed to confirm the toxicity of lincomycin in $\mathrm{HaCaT}$ cells, which are related to melanocytes. As shown in Fig. 1C, there were no significant effects on cell viability in $\mathrm{HaCaT}$ cells treated with lincomycin $(0.5$ and $1 \mathrm{mg} / \mathrm{mL})$, relative to the untreated control cells.

Effect of lincomycin on melanin production and intracellular tyrosinase activity

To examine the effect of lincomycin on melanin synthesis, melanin content, and tyrosinase activity, tests were performed. The B16F10 cells were treated with the indicated concentrations of lincomycin for $72 \mathrm{~h}$. $\alpha$-melanocyte stimulating hormone (MSH) $(200 \mathrm{nM})$ was used as the positive control. As shown in Fig. 2A, the melanin content increased in a concentration-dependent manner. In particular, at a concentration of $2 \mathrm{mg} / \mathrm{mL}$, the melanin content dramatically increased to $370 \%$ compared with the untreated control cells, and it was $132 \%$ higher than that of the positive control group. To clarify whether lincomycin affects melanogenesis via tyrosinase activity, we performed an intracellular tyrosinase activity test. As shown in Fig. 2B, tyrosinase activity was increased by lincomycin and $\alpha$-MSH treatments at the indicated concentrations compared with that of the untreated control cells. In particular, at the highest concentration of lincomycin, tyrosinase activity was increased by $750 \%$ compared with the untreated control cells, and this was $214 \%$ higher than that in the positive control group.
Effect of lincomycin on the expression of melanogenic enzymes and MITF in B16F10 cells

To determine whether lincomycin regulates the expression of melanogenic enzymes, such as tyrosinase and TRP1/2, B16F10 cells were treated with lincomycin $(0.5,1$, and $2 \mathrm{mg} / \mathrm{mL})$ for $43 \mathrm{~h}$. The protein expressions of the melanogenic enzymes were examined using Western blotting. Compared with the untreated control cells, lincomycin treatment at concentrations ranging from 0.5 to $2 \mathrm{mg} /$ $\mathrm{mL}$ significantly increased the melanogenic enzyme levels in a concentration-dependent manner (Fig. 3). To clarify the transcriptional regulation of melanogenic enzyme activation, we evaluated the effects of lincomycin on MITF expression. MITF expression significantly increased in a concentration-dependent manner (Fig. 4). Thus, the results suggested that lincomycin induces melanogenic enzyme transcription by increasing the MITF expression levels.

\section{Effect of lincomycin on MAPK phosphorylation in B16F10 cells}

Recent studies have reported that MAPK phosphorylation regulates MITF expression, thereby regulating melanogenesis [13-15]. Thus, to confirm the effect of lincomycin on phosphorylation in the upstream pathway in melanogenesis, Western blot analysis was performed on the B16F10 cells treated with the indicated concentrations. In the MAPK family, lincomycin significantly increased p38 phosphorylation (Fig. 5), but did not have a significant effect on JNK and ERK phosphorylation (data not shown). To determine whether p38 phosphorylation are involved in the upregulation of tyrosinase activity, we treated the B16F10 cells with SB203580 (a specific inhibitor of p38). As shown in Fig. 6, the tyrosinase activity induced in B16F10 cells using lincomycin treatment was reduced by treating them with the inhibitors, suggesting that the p38 MAPK signaling pathway is 
(A)
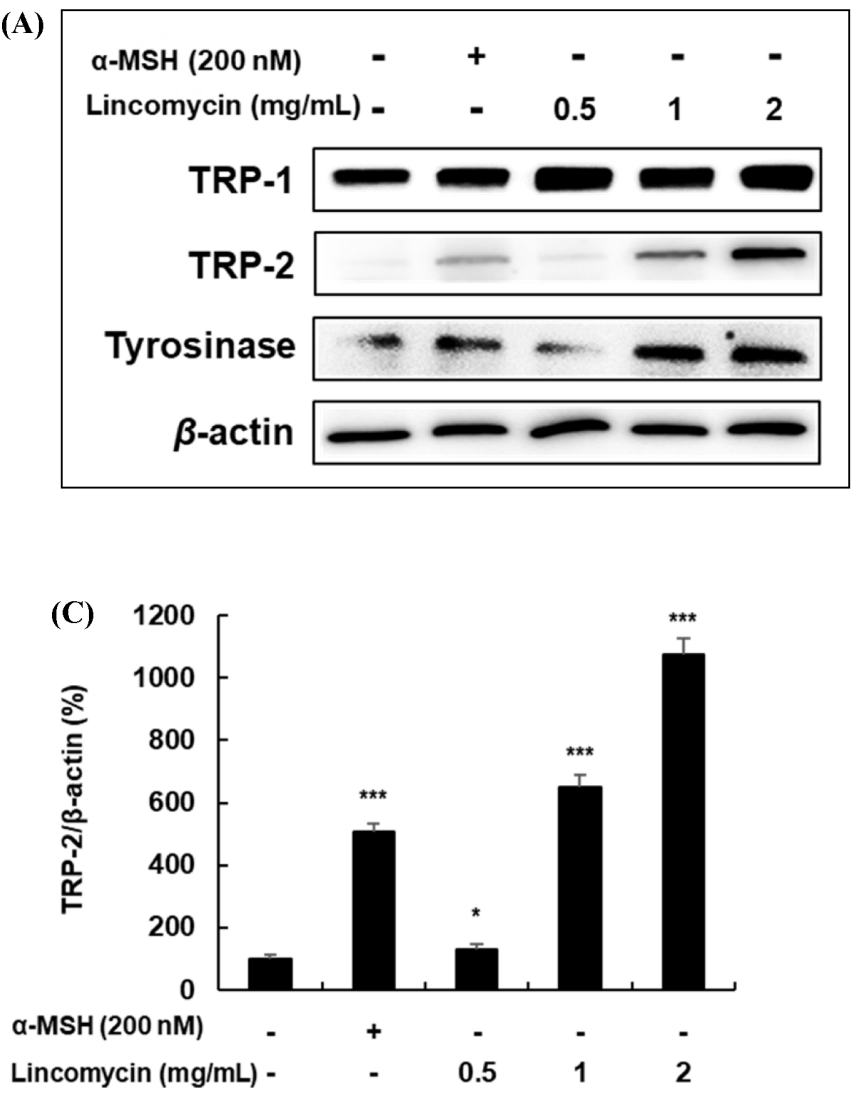

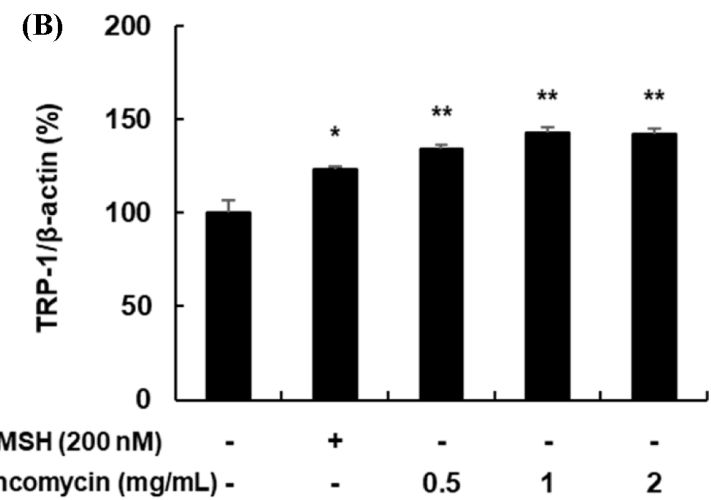

Lincomycin $(\mathrm{mg} / \mathrm{mL})-\quad \quad-\quad 0.5 \quad 1 \quad 2$

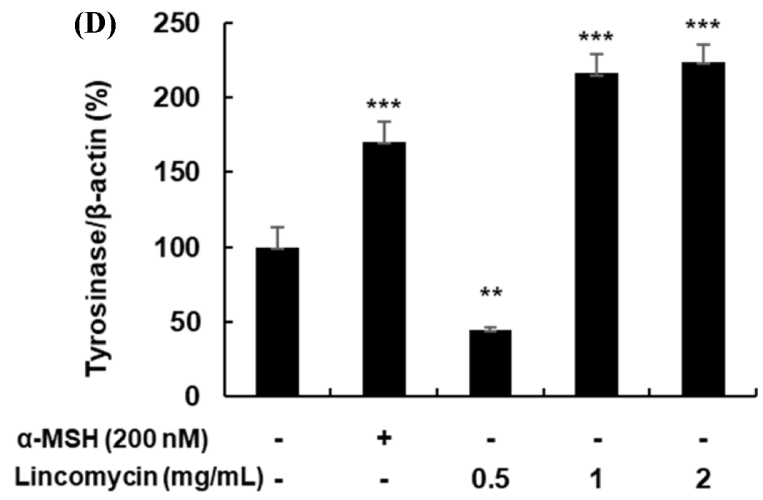

Fig. 3 Effect of lincomycin on the expression of melanogenic enzymes in B16F10 cells. The cells were treated with various concentrations of lincomycin $(0.5,1$, and $2 \mathrm{mg} / \mathrm{mL}$ ) for 43 h. Protein levels were evaluated using Western blot analysis. (A) Results of Western blotting and protein levels of (B) TRP-1, (C) TRP-2, and (D) tyrosinase. Results are expressed as percentages compared to the untreated control cells. The results of all data are expressed as the mean \pm standard deviation ( $\mathrm{SD}: \mathrm{n}=4$ ) of at least four independent experiments. ${ }^{*} p<0.05,{ }^{* *} p<0.01,{ }^{* * *} p<0.001$ vs. untreated control cells. TRP: tyrosinase-related protein. $\alpha$-MSH: positive control

(A)

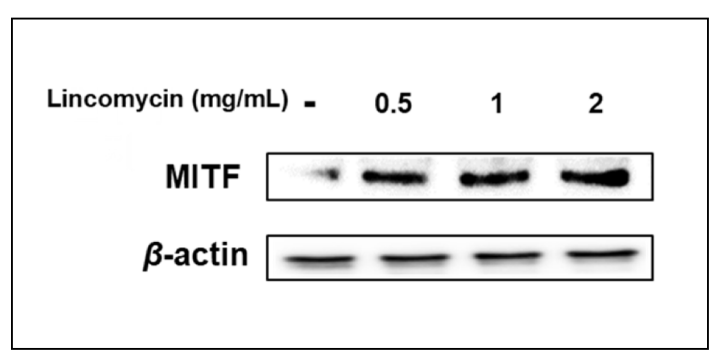

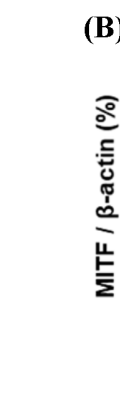

Lincomycin $(\mathrm{mg} / \mathrm{mL})$

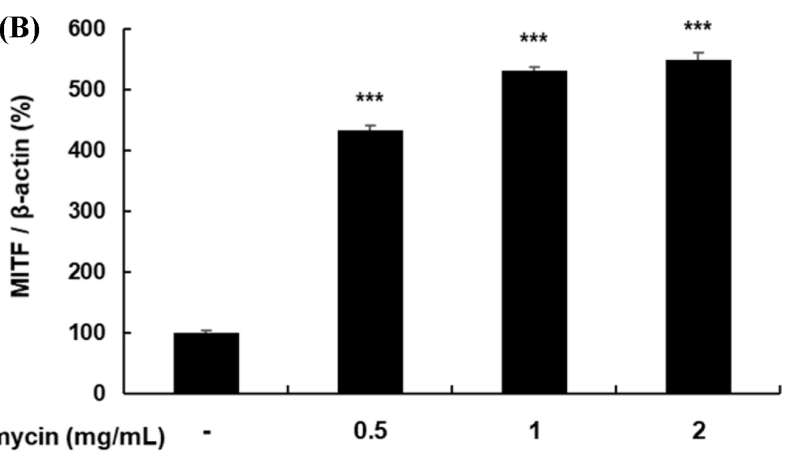

Fig. 4 Effect of lincomycin on MITF expression in B16F10 cells. The cells were treated with various concentrations of lincomycin (0.5, 1 , and 2 mg/ $\mathrm{mL}$ ) for $24 \mathrm{~h}$. (A) Results of Western blotting, and (B) protein levels of MITF expression, which were quantified using image J program. Results are expressed as a percentage of the untreated control cell. The results of all data are expressed as the mean \pm standard deviation (SD: $\mathrm{n}=4$ ) of at least four independent experiments. $* * * p<0.001$ vs. untreated control cells. MITF: microphthalmia-associated transcription factor, SD: standard deviation

directly involved in melanogenesis.

\section{Effect of lincomycin on PKA signaling pathway}

PKA moves into the nucleus of melanocytes to increase the phosphorylation of CREB, and the phosphorylated CREB binds to the CRE in the MITF promoter to induce the expression of MITF [16-18]. To understand whether the PKA signaling pathway is involved in lincomycin-stimulated melanogenesis, we examined the effect of H-89 (a specific PKA inhibitor) on tyrosinase activity. As shown in Fig. 7, the tyrosinase expression was 
(A)

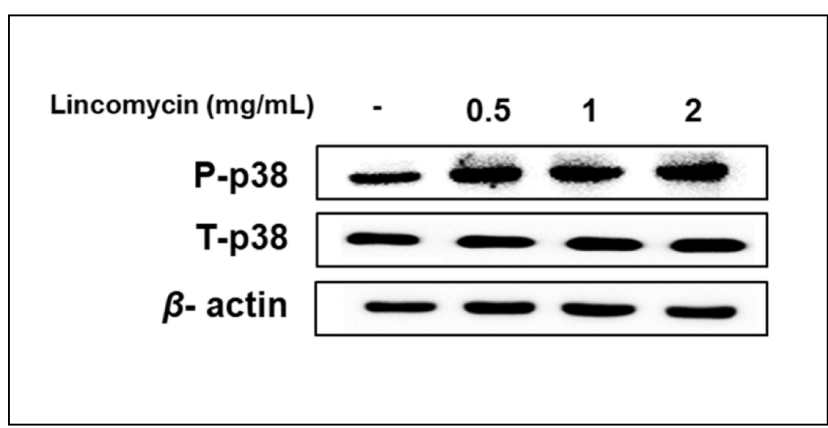

(B)

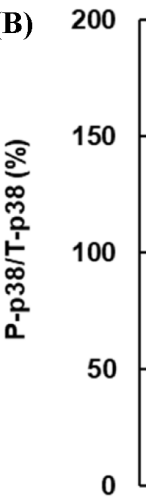

Lincomycin $(\mathrm{mg} / \mathrm{mL})$

0

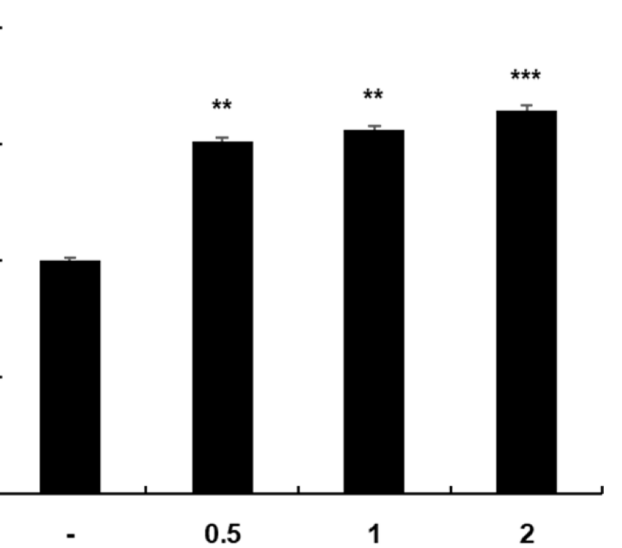

Fig. 5 Effects of lincomycin on p-38 expression. The cells were treated with lincomycin at the indicated concentrations for 20 h. (A) Results of Western blotting and protein levels of (B) p-p38. The results of all data are expressed as the mean \pm standard deviation (SD: $n=4)$ of at least four independent experiments. ${ }^{*} p<0.05,{ }^{* *} p<0.01,{ }^{* * *} p<0.001$ vs. untreated control cells. P: Phosphorylated; SD: standard deviation; T: Total

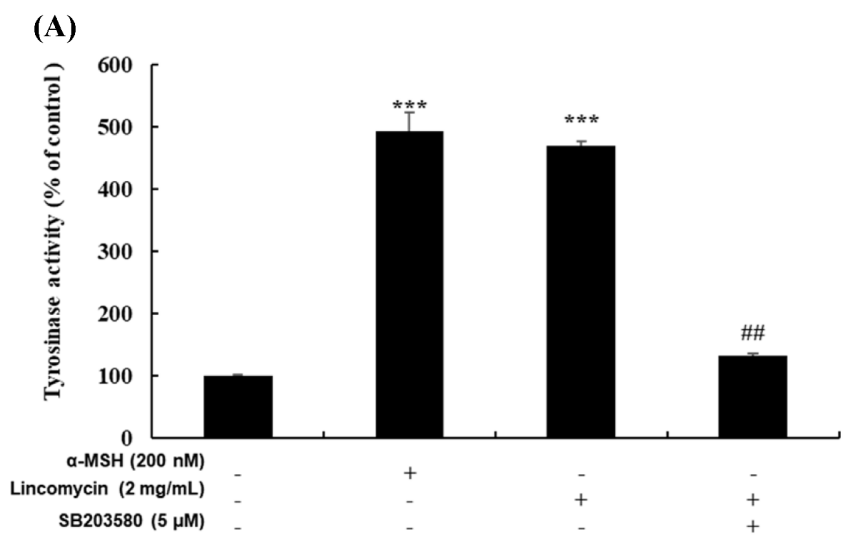

(B)
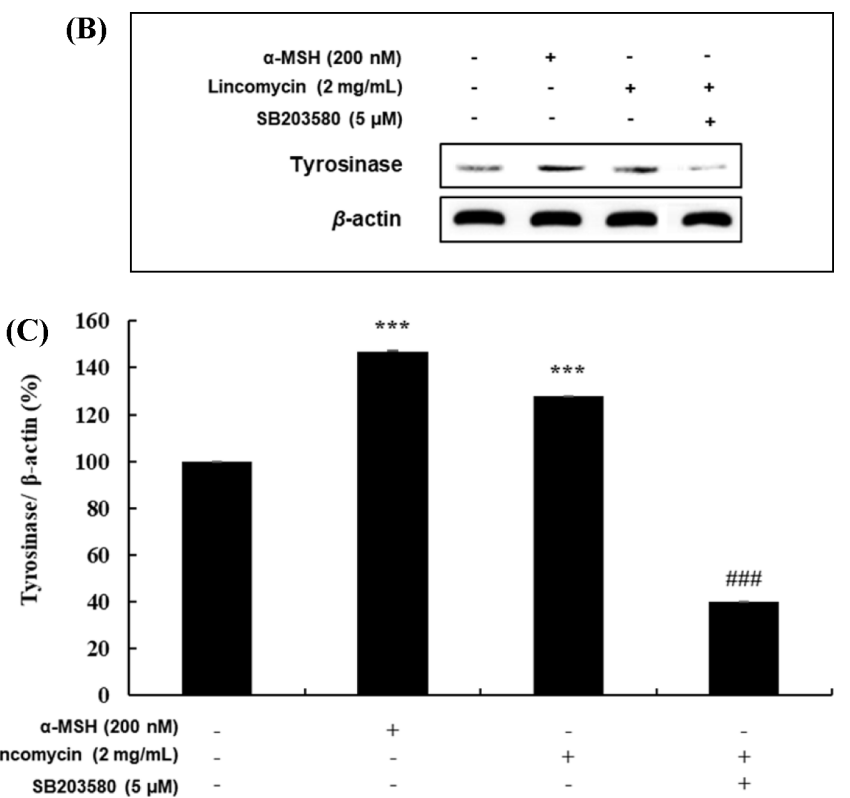

Fig. 6 Effect of MAPK inhibitor on lincomycin-induced tyrosinase activity in B16F10 cells. To understand the involvement of lincomycin on MAPK signaling pathway in melanogenesis, (A) a cellular tyrosinase activity assay and (B) Western blotting were performed using the following MAPK inhibitors: SB203580 (p38 inhibitor). (C) The protein levels of tyrosinase were quantified using image J program. The results of all data are expressed as the mean \pm standard deviation (SD: $\mathrm{n}=4$ ) of at least four independent experiments. ${ }^{* *} p<0.01,{ }^{* *} p<0.001$, vs. untreated control cells, and

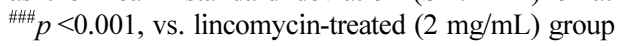

reduced when treated with lincomycin and H-89, compared with the lincomycin treatment alone. These results suggest that lincomycin may be related to the PKA signaling pathway in inducing tyrosinase activation in the cells.

\section{Effect of lincomycin on AKT signaling pathway}

The inhibition of PI3K/AKT phosphorylation has been reported to be involved in activating melanogenesis in human melanocytes and B16F10 melanoma cells by reducing MITF phosphorylation, preventing the degradation of MITF [19-22]. To determine the effect of lincomycin on the AKT pathway in B16F10 cells, we performed Western blot analysis. As shown in Fig. 8, AKT phosphorylation was reduced by treating cells with lincomycin, compared with the untreated control cells.

\section{Discussion}

Drug development by drug repositioning is a strategy for identifying new uses of approved or developing drugs outside the scope of their original medical indications. This strategy offers several advantages over developing an entirely new drug for a 
(B)
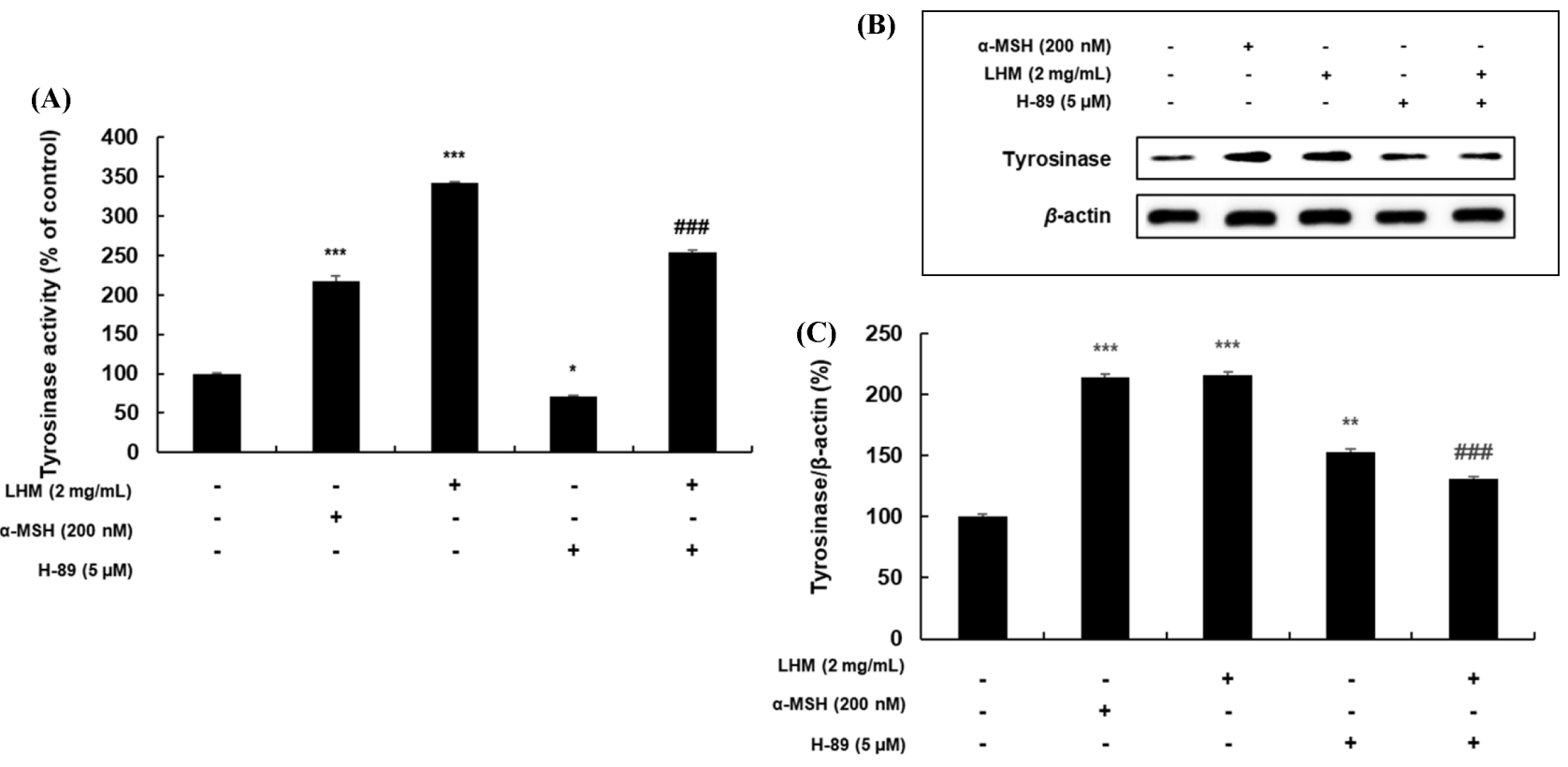

Fig. 7 Effect of PKA inhibitor on lincomycin-induced tyrosinase activity in B16F10 cells. To understand the involvement of lincomycin on PKA signaling pathway in melanogenesis, (A) a cellular tyrosinase activity and (B) Western blotting were performed using the following PKA inhibitor: H89 (PKA inhibitor). (C) The protein levels of tyrosinase were quantified using image $\mathrm{J}$ program. The results of all data are expressed as the mean \pm standard deviation (SD: $\mathrm{n}=4$ ) of at least four independent experiments. ${ }^{*} p<0.05,{ }^{* *} p<0.01,{ }^{* * *} p<0.001$, vs. untreated control cells and ${ }^{\# \#} p<0.001$, vs. lincomycin -treated $(2 \mathrm{mg} / \mathrm{mL})$ group

(A)

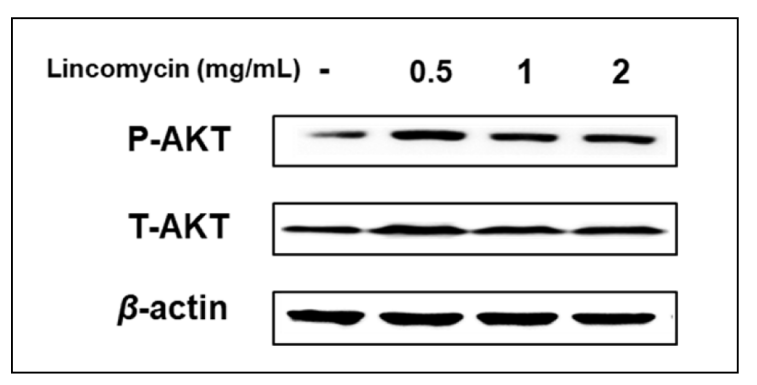

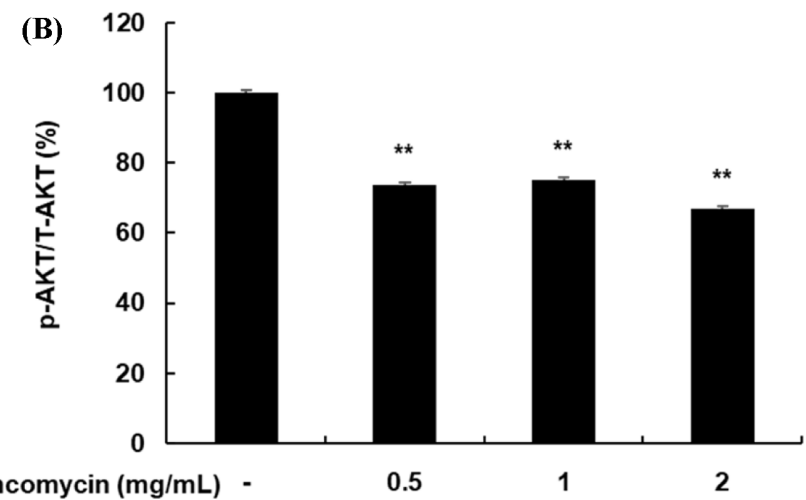

Fig. 8 Effects of lincomycin on phosphorylation of AKT. The cells were treated with lincomycin $(0.5,1$, and $2 \mathrm{mg} / \mathrm{mL})$ for $24 \mathrm{~h}$. (A) Results of Western blotting and protein levels of (B) P-AKT. Results are expressed as a percentage of the control. The results of all data are expressed as the mean \pm standard deviation (SD: $\mathrm{n}=4$ ) of at least four independent experiments. ${ }^{* *} p<0.01$ vs. untreated control cells. P: Phosphorylated; SD: standard deviation; T: Total

given indication [33,34]. For several years, our research team has studied the pathways involved in regulating pigmentation by treating melanocytes with specific substances or old antibiotics. It has also been suggested that these compounds or old antibiotics may be used as therapeutic or cosmetic agents [27,28].

The most important role of melanin in the mammalian epidermis is to prevent from skin damage against UV radiation, toxic drugs and chemicals. In addition, the quantity of melanin present determines the coloration of the skin, eyes, and hair. Consequently, a lack of melanogenesis leads to hypopigmentation and increases the risk of skin cancer and of various skin depigmentation diseases, such as achromic naevus vitiligo, and albinism [35,36]. In this study, we evaluated the effect of lincomycin on the melanogenic activity and molecular mechanism in mouse B16F10 melanoma cells. To determine the effect of lincomycin on melanogenesis in B16F10 cells, melanin synthesis and tyrosinase activity tests were performed. In addition, Western blot analysis was performed to confirm the expression of tyrosinase, TRP1, and TRP2, as well as of their regulator, MITF. Moreover, Western blotting was used to determine the effect of lincomycin on the phosphorylation in MAPK, PI3K/AKT and PKA signaling pathways, which are regulators of MITF expression. 
The results of our study showed that lincomycin stimulated melanogenesis in B16F10 cells.

An MTT assay was performed to determine the concentration of lincomycin that was not cytotoxic. The results showed that lincomycin exerted cytotoxicity at a concentration of $4 \mathrm{mg} / \mathrm{mL}$, but it did not at a concentration of $0.25,0.5,1$, and $2 \mathrm{mg} / \mathrm{mL}$ (Fig. 1). Thus, these concentrations were used for the subsequent experiments. In addition, lincomycin treatment of B16F10 cells increased the melanin synthesis and intracellular tyrosinase activity in a concentration-dependent manner (Fig. 2).

Recent researches have reported that melanogenesis is controlled by the complex enzymatic cascade of melanogenic enzymes (TRP1, 2, and tyrosinase) that are regulated by MITF [11,12]. Therefore, the activation of MITF expression promotes melanogenic enzyme transcription, thereby upregulating melanogenesis in B16F10 cells. The results of our study indicated an increase in the expression of MITF and of melanogenic enzymes in the cells after lincomycin treatment, in a concentration-dependent manner (Figs. $3,4)$. These results suggested that lincomycin stimulates MITF expression, thereby increasing the expression of melanogenic enzymes, and leading to an up-regulation of melanogenesis.

It has been proposed that the MAPK/AKT signaling pathways are involved in melanogenesis in B16F10 cells [13-15, 19-22]. The stimulation of JNK and p38 phosphorylation upregulates melanogenesis by activating MITF expression and melanogenic enzyme transcription in the cells. However, the activation of ERK and AKT phosphorylation increases MITF phosphorylation, leading to its degradation, thereby downregulating melanogenesis. In this study, lincomycin increased the phosphorylation of p38 and decreased AKT phosphorylation but did not significantly affect the phosphorylation of JNK and ERK (Figs. 5, 7). Thus, to determine whether the p38 signaling pathway is involved in melanogenesis, specific inhibitor of p38 (SB203580) were used to treat cells. As shown in Fig. 6, tyrosinase activity was stimulated by $\alpha$-MSH and lincomycin, but due to p38 pathway inhibitory action of the inhibitors, the tyrosinase activity was attenuated in the inhibitors and lincomycin group, compared to the $\alpha-\mathrm{MSH}$ and lincomycin groups. These results indicate that lincomycin is directly involved in melanogenesis by activating the expression of tyrosinase through the p38 MAPK and PI3K/AKT signaling pathways.

Previous researches have reported that the cAMP/PKA signaling pathway is associated with melanogenesis. cAMP activates PKA and this activated form moves into the nucleus of melanocytes to increase the phosphorylation of CREB, and activates the MITF promoter to induce the expression of MITF, resulting in melanogenesis via an increased expression of melanogenic-related proteins [1618]. To understand the PKA signaling pathway, H-89 (an inhibitor of PKA) was used for treatment and a tyrosinase activity test was performed. Our results showed that the tyrosinase activity was inhibited by lincomycin and H-89 treatment, compared to lincomycin treatment alone, suggesting that lincomycin may be related to the PKA signaling pathway in inducing tyrosinase activation in the cells (Fig. 8).

To summarize, this work is the first to evaluate the melanogenesis effect of lincomycin, which was found to stimulate melanogenesis in B16F10 melanoma cells. The data of our study showed that lincomycin induced p38 phosphorylation and suppressed the phosphorylation of AKT via MAPK and PI3K/AKT signaling pathways, which resulted in MITF and melanogenic enzyme activation, thereby increasing melanogenesis. Therefore, these results suggest that the new efficacy of lincomycin, which is not much in used. Although further research is needed on the efficacy and safety of lincomycin in clinical studies, lincomycin could be used to treat hypopigmented skin diseases such as albinism and vitiligo.

Acknowledgments This research was supported by the Ministry of Trade, Industry \& Energy (MOTIE), Korea Institute for Advancement of Technology (KIAT) through the Construction Project for Skin Clinical Solution Center (P0017663).

\section{References}

1. Hearing VJ (2005) Biogenesis of pigment granules: A sensitive way to regulate melanocyte function. J Dermatol Sci 37: 3-14. doi: 10.1016/ j.jdermsci.2004.08.014

2. Tadokoro T, Yamaguchi, Y, Batzer J, Coelho SG, Zmudzka BZ, Miller SA, Wolber R, Beer JZ, Hearing VJ (2005) Mechanisms of skin tanning in different racial/ethnic groups in response to ultraviolet radiation. $\mathrm{J}$ Investig Dermatol 124: 1326-1332. doi: 10.1111/j.0022-202X.2005.23760.x

3. Brenner M, Hearing VJ (2008) The protective role of melanin against UV damage in human skin. Photochem Photobiol 84: 539-549. doi: 10.1111/j.1751-1097.2007.00226.x

4. Bonaventure J, Domingues MJ, Larue L (2013) Cellular and molecular mechanisms controlling the migration of melanocytes and melanoma cells. Pigment Cell Melanoma Res 26: 316-325. doi: 10.1111/pcmr.12080

5. Tabassum N, Hamdani M (2014) Plants used to treat skin diseases. Pharmacogn Rev 8: 52-60. doi: 10.4103/0973-7847.125531

6. d'Ischia M, Wakamatsu K, Cicoira F, Mauro ED, Garcia-Borron JC, Commo S, Galvan I, Ghanem G, Kenzo K, Meredith P, Pezzella A, Santato C, Sarna T, Simon JD, Zecca L, Zucca FA, Napolitano A, Ito S (2015) Melanins and melanogenesis: From pigment cells to human health and technological applications. Pigment Cell Melanoma Res 28: 520-544. doi: 10.1111/pcmr.12393

7. Hearing VJ, Jimenez M (1987) Mammalian tyrosinase-the critical regulatory control point in melanocyte pigmentation. Int J Biochem 19: 1141-1147. doi: 10.1016/0020-711x(87)90095-4

8. Tsukamoto K, Jackson IJ, Urabe K, Montague PM, Hearing VJ (1992) A second tyrosinase-related protein, TRP2, is a melanogenic enzyme termed DOPAchrome tautomerase. EMBO J 11: 519-526

9. Jiménez-Cervantes C, Solano F, Kobayashi T, Urabe K, Hearing VJ, Lozano JA, Garcia-Borrón JC (1994) A new enzymatic function in the melanogenic pathway. The 5,6-dihydroxyindole-2-carboxylic acid oxidase activity of tyrosinase-related protein-1 (TRP1). J Biol Chem 269: 17993-18000

10. Sugumaran M (2016) Reactivities of quinone methides versus oquinones in catecholamine metabolism and eumelanin biosynthesis. Int $\mathrm{J}$ Mol Sci 17: 1576. doi: 10.3390/ijms17091576

11. Bentley NJ, Eisen T, Goding CR (1994) Melanocyte-specific expression of the human tyrosinase promoter: activation by the microphthalmia 
gene product and role of the initiator. Mol Cell Biol 14: 7996-8006. doi: 10.1128/mcb.14.12.7996-8006.1994

12. Vachtenheim J, Borovansky J (2010) "Transcription physiology" of pigment formation in melanocytes: central role of MITF. Exp Dermatol 19: 617-627. doi: 10.1111/j.1600-0625.2009.01053.x

13. Widlude HR, Fisher DE (2003) Microphthalmia-associated transcription factor: A critical regulator of pigment cell development and survival. Oncogene 22: 3035-3041. doi: 10.1038/sj.onc.1206443

14. Saha B, Singh SK, Sarkar C, Bera R, Ratha J, Tobin DJ, Bhadra R (2006) Activation of the Mitf promoter by lipid-stimulated activation of p38-stress signaling to CREB. Pigment Cell Res 19: 595-605. doi: 10.1111/j.1600-0749.2006.00348.x

15. Ahn JH, Jin SH, Kang HY (2008) LPS induces melanogenesis through p38 MAPK activation in human melanocytes. Arch Dermatol Res 300: 325-329. doi: 10.1007/s00403-008-0863-0

16. Kim A, Yim NH, Im M, Jung YP, Liang C, Cho WK, Ma JY (2013) Ssanghwa-tang, an oriental herbal cocktail, exerts anti-melanogenic activity by suppression of the p38 MAPK and PKA signaling pathways in B16F10 cells. BMC Complement Altern Med 13: 214. doi: 10.1186/ 1472-6882-13-214

17. Chan CF, Huang CC, Lee MY, Lin YS (2014) Fermented broth in tyrosinase-and melanogenesis inhibition. Molecules 19: 13122-13135. doi: 10.3390/molecules190913122

18. Sun M, Xie HF, Tang Y, Lin SQ, Li JM, Sun SM, Hu XL, Huang YX, Shi W, Jian D (2017) G protein-coupled estrogen receptor enhances melanogenesis via cAMP-protein kinase (PKA) by upregulating microphthalmia-related transcription factor-tyrosinase in melanoma. J Steroid Biochem Mol Bio. 165: 236-246. doi: 10.1016/j.jsbmb.2016.06.012

19. Kim HJ, Kim IS, Dong Y, Lee IS, Kim JS, Kim JS, Woo JT, Cha BY (2015) Melanogenesis-inducing effect to cirsimaritin through increases in microphthalmia-associated transcription factor and tyrosinase expression. Int J Mol Sci 16: 8772-8788. doi: 10.3390/ijms16048772

20. Yao C, Jin CL, Oh JH, Oh IG, Park CH, Chung JH (2015) Ardisia crenata extract stimulates melanogenesis in B16F10 melanoma cells through inhibition ERK1/2 and Akt activation. Mol Med Rep 11: 653657. doi: $10.3892 / \mathrm{mmr} .2014 .2697$

21. Chung YC, Kim S, Kim JH, Lee GS, Lee JN, Lee NH, Hyun CG (2017) Pratol, an O-methylated flavone, induces melanogenesis in B16F10 melanoma cells via p-p38 and p-JNK upregulation. Molecules 22: 1704. doi: 10.3390/molecules22101704

22. Im DS, Lee JM, Lee J, Shin HJ, No KT, Park SH, Kim K (2017) Inhibition of collagenase and melanogenesis by ethanol extracts of Orostachys japonicus A. Berger: Possible involvement of Erk and Akt signaling pathways in melanoma cells. Acta Biochim Biophys Sin 49:
945-953. doi: 10.1093/abbs/gmx090

23. MacLeod AJ, Ross HB, Ozere RL, Digout Geo, van Rooyen CE (1964) Lincomycin: A New Antibiotic Active Against Staphylococci and Other Gram-Positive Cocci. Can Med Assoc J 91: 1056-1060

24. Duncan IBR, Jeans B (1965) Lincomycin in Hospital Practice. Can Med Assoc J 93: 685-691

25. Phillips I (1981) Past and current use of clindamycin and lincomycin. J Antimicrob Chemother 7: 11-18. doi: 10.1093/jac/7.suppl_a.11

26. Wrześniok D, Otręba M, Beberok A, Buszman E (2013) Impact of kanamycin on melanogenesis and antioxidant enzymes activity in melanocytes-an in vitro study. J Cell Biochem 114: 2746-2752. doi: $10.1002 / \mathrm{jcb} .24623$

27. Moon SH, Chung YC, Hyun CG (2019) Tobramycin promotes melanogenesis by upregulating p38 MAPK protein phosphorylation in B16F10 melanoma cells. Antibiotics 8: 140. doi: 10.3390/antibiotics 8030140

28. Ullah S, Chung YC, Hyun CG (2020) Induction of melanogenesis by fosfomycin in B16F10 cells through the upregulation of P-JNK and Pp38 signaling pathways. Antibiotics 9: 172. doi: 10.3390/antibiotics9040172

29. Seiberg M (2001) Keratinocyte-melanocyte interactions during melanosome transfer. Pigment Cell Res 14: 236-242. doi: 10.1034/ j.1600-0749.2001.140402.x

30. Lin JY, Fisher DE (2007) Melanocyte biology and skin pigmentation. Nature 445: 843-850. doi: 10.1038/nature05660

31. Delevoye C (2014) Melanin transfer: The keratinocytes are more than gluttons. J Investig Dermatol 134: 877-879. doi: 10.1038/jid.2013.487

32. Amico-Ruvio SA, Paganelli MA, Myers JM, Popescu GK (2012) Ifenprodil effects on GluN2B-containing glutamate receptors. Mol Pharmacol 82: 1074-1081. doi: 10.1124/mol.112.078998

33. Pushpakom S, Iorio F, Eyers PA, Escott KJ, Hopper S, Wells A, Doig A, Guilliams T, Latimer J, McNamee C, Norris A, Sanseau P, Cavalla D, Pirmohamed M (2019) Drug repurposing: progress, challenges and recommendations. Nat Rev Drug Discov. 18: 41-58. doi: 10.1038/ nrd.2018.168

34. Jeong SM, Yoon TJ (2021) Development of Pigmentation-Regulating Agents by Drug Repositioning. Int J Mol Sci 22: 3894. doi: 10.3390/ ijms22083894

35. Briganti S, Camera E, Picardo M (2003) Chemical and instrumental approaches to treat hyperpigmentation. Pigment Cell Res 16: 101-110. doi: 10.1034/j.1600-0749.2003.00029.x

36. Miyamura YI, Coelho SG, Wolber R, Miller SA, Wakamatsu K, Zmudzka BZ, Ito S, Smuda C, Passeron T, Choi W (2007) Regulation of human skin pigmentation and responses to ultraviolet radiation. Pigment Cell Res 20: 2-13. doi: 10.1111/j.1600-0749.2006.00358.x 